Relations industrielles

Industrial Relations

\title{
Leadership Dynamics and the Trade-Union Leader. Lois MacDonald and Associates. N.Y. New York University Press, 1959, 150 pp.
}

\section{C.-R. Giroux}

Volume 15, numéro 2, avril 1960

URI : https://id.erudit.org/iderudit/1022047ar

DOI : https://doi.org/10.7202/1022047ar

Aller au sommaire du numéro

Éditeur(s)

Département des relations industrielles de l’Université Laval

ISSN

0034-379X (imprimé)

1703-8138 (numérique)

Découvrir la revue

Citer ce compte rendu

Giroux, C.-R. (1960). Compte rendu de [Leadership Dynamics and the

Trade-Union Leader. Lois MacDonald and Associates. N.Y. New York University Press, 1959, 150 pp.] Relations industrielles / Industrial Relations, 15(2), 283-285. https://doi.org/10.7202/1022047ar

Tous droits réservés (C Département des relations industrielles de l’Université Laval, 1960
Ce document est protégé par la loi sur le droit d'auteur. L'utilisation des services d'Érudit (y compris la reproduction) est assujettie à sa politique d'utilisation que vous pouvez consulter en ligne.

https://apropos.erudit.org/fr/usagers/politique-dutilisation/ 
interaction. Le eleader》 et les groupes peuvent être motivés soit à résoudre des problèmes «Task Oriented 》, soit à satisfaire leurs propres besoins personnels « Self Oriented ». Le «leader $\gg$ efficace sera celui qui pourra diriger le groupe vers une action créatrice dans la solution des problèmes communs. Par son comportement personnel il accondera aux membres de la reconnaissance pour leur performance et réduira ainsi les possibilités de punition.

Les membres qui auront expérimenté un certain succès avec un \&leader » auront tendance à suivre cette même personne dans l'avenir. De même, un «leader efficace aura plus de chances de réussir dans d'autres situations semblables et son succès passé l'amènera à essayer de conduire le groupe quand les circonstances l'exigeront. Les expé riences rapportées par Bass semblent indiquer que le membre le plus capable deviendra tout probablement le «leader $\gg$ du groupe. Si ce membre a l'habileté nécessaire pour résoudre les problèmes du groupe, il semble qu'il réussira à convaincre les membres à accepter son autorité. Il semble que certains membres en vertu de leur position ou de leurs qualités personnelles sont en mesure de récompenser ou de punir directement les autres membres du groupe. Un individu jouissant d'un rang élevé, qui lui permet de récompenser ou de désapprouver l'action du groupe, peut forcer le groupe à atteindre un but déterminé. Nous faisons face à ce moment là à une autorité qui devient facilement absolue et parfois même arbitraire. Si toutefois la récompense ou la punition est déterminée par le groupe ou est utilisée prudemment, le «leader 》, même jouissant d'une autorité hiérarchique exercera alons une action permissive. Notons toutefois que la coercion et la permission requièrent toutes deux le pouvoir délégué par une autorité hiéranchique. Dans un groupe démocratique, une telle autorité étant accordée au «leader » par les membres du groupe, il ne peut pas utiliser la coercion. Nous constatons donc que le conformisme ne peut exister qu'en fonction d'une interaction basée sur une hiérarchie bien structurée. Le prestige attaché à une position, et l'estime individuel, sont tous deux source d'habileté et de pouvoir. Quand le prestige et l'estime ne concordent pas les conflits surgissent facilement. Les deux dernières parties du volume traitent de divers attributs du groupe: le nombre des membres, la proximité entre eux, l'estime mutuel, les communications, la familiarité, l'homogénéité et les autres variables qui peuvent augmenter la possibilité d'interaction entre les membres. Tout changement dans la possibilité d'interaction affecte directement l'efficacité du groupe. Finalement l'auteur indique diverses méthodes pour augmenter l'efficacité d'un groupe.

La plupart des énoncés précédents. sont corroborés par des résultats expérimentaux. Toutefois, plusieurs restent encore à être démontrés de façon pratique.

Bass n'a pas la prétention d'élaborer une théorie définitive. Comme il le dit lui-même: «Paradoxically, a satisfactory theory begins to be modified almost as soon as it is cncluded: an inadequate one more often lasts unchanged for centuries. The goal of simplicity is congruent with the purpose of theorybuilding: to promote understanding $>$. Ceci se rapproche du fameux mot de J.J. Thomas: \& A theory is a policy rather than a creed $\gg$.

Cette oeuvre de Bass nous permet d'entrevoir les motivations en jeu dans la dynamique du groupe. Maintenant, il nous reste à tout mettre en pratique.

Cette brillante synthèse de Bass va certainement stimuler la recherche sur la dynamique et la structure des groupes.

$$
\text { C.-R. Giroux }
$$

Leadership Dynamics and the TradeUnion Leader. Lois MacDonald and Associates. N.Y. New York University Press, $1959,150 \mathrm{pp}$.

La croissance phénoménale des unions ouvrières depuis environ quinze ans a fait surgir une foule de problèmes d'interrelations de groupes et de nombreux conflits d'autorité. Une organisation interne inadéquate, l'incompétence et l'incompréhension des représentants syndicaux furent souvent à l'origine de frictions non seulement entre le capital et lo travail mais entre les membres eux- 
mêmes et leurs propres meneurs.

Afin d'analyser le problème complexe de l'autorité syndicale, MacDonald et ses associés font un relevé complet des recherches sur le «leadership 》 syndical. Par suite de l'intérêt croissant pour les problèmes administratifs, la structure des organisations industrielles et les caractéristiques des «leaders », i.e. des exécutifs, il était essentiel de vérifier si les unions ouvrières avaient été l'objet de recherches identiques. Toute recherche sur la croissance des unions ouvrières doit considérer certaines questions bien spécifiques. Quelle est la personnalité des hommes qui recherchent les postes de commande? Quelles méthodes utilisent-ils pour obtenir et maintenir leurs positions? Leurs caractéristiques et leurs techniques changent-elles à mesure qu'ils s'élèvent dans la hiérarchie et à mesure que la puisance et l'influence des unions s'accroissent? Quels facteurs dirigent l'évolution vers une autorité saine et démocratique ou vers une autorité dominatrice et corrompue? Jusqu'à quel point la «professionnalisation 》 des postes administratifs a-t-elle affecté le comportement du «leader»? Dans quelle mesure l'embauchage de spécialistes a-t-elle limité l'autorité administrative? Voilà autant de questions auxquelles l'auteur cherche des réponses dans son analyse des publications.

L'auteur relève trois orientations différentes dans les techniques utilisées pour analyser le fonctionnement des syndicats: l'approche microscopique, l'approche macroscopique et l'approche électique. Dans l'orientation MICROSCOPIQUE, les recherches portent sur de petits groupes comprenant souvent moins de douze personnes ou de petits segments de l'organisation: 1) L'ECOLE DES RELATIONS HUMAINES: Elle s'intéresse à l'organisation de l'activité du groune afin d'obtenir des résultats plus productifs par la reconnaissance de l'impact des relations personnelles. 2) LA DYNAMIOUE DE GROUPE: quoique semblable à l'approche des relations humaines, cette école considère surtout les processus d'interactions entre les meneurs et les membres. 3) J.A SOCIEMETRIE mesure les relations interpersonnelles à partir des attitudes et des réponses des membres. Elles essaient d'évaluer les qualités du "leadershin » et son acceptabilité. 4) LA RECHER-
CHE SUR LES PETITS GROUPES ET LES GROUPES SANS \&LEADER * étudient le comportement des membres au sein de groupes non-structurés, afin de découvrir comment émergent les «leaders 》 et comment les membres répondent aux situations demandant l'intervention d'un «leader». 5) Les recherches de l'Université de l'Ohio se concentrent surtout sur les problèmes du "leadership », sur les rôles du meneur et sur les perceptions impliquées dans le milieu du travail. La majorité des études microscopiques sont viciées soit parce qu'elles sont trop anecdotiques, soit parce qu'elles se basent sur un trop petit nombre de sujets, soit parce qu'elles représentent des situations de laboratoire.

La deuxième classification des recherches résulte d'une orientation macroscopique. Ces études portent surtout sur de larges organisations qui jouissent de structures formelles et d'un modus operandi clairement défini. Les psychologues sociaux considèrent les questions du «leadership » avec plus d'envergure: l'évaluation et les caractéristiques des meneurs, les processus d'interactions entre les meneurs et les membres. Ces recherches macroscopiques sont basées sur la nature des structures, la puissance de la hiérarchie, l'exercice de l'autorité, les systèmes de communications et le développement de la bureaucratie. Le syndicalisme en refute plusieurs parce qu'elles manquent d'objectivité et qu'elles utilisent des méthodes mises à point pour analyser les institutions industrielles et commerciales.

La troisième catégorie groupe des travaux divers: études sur les représentants svndicaux, les bibliographies, les études de cas en relations industrielles. Ces travaux sont surtout descriptifs et ne pèchent certes pas par excès de rigueur scientifique.

Il ne faut pas s'étonner si les recherches sur les unions ouvrières sont restreintes en quantité et en qualité. En efftet, l'on commence à peine à analyser et à comprendre l'organisation industrielle et le comportement des gérants et des exécutifs. Il n'existe même pas une philosophie ou une théorie du «leadership » industriel.

Dans son dernier chapitre l'auteur revoit la situation et suggère des points 
de recherche très importants. 1)L'école des relations humaines pourra étudier les motivations des représentants syndicaux. Toutefois, elle doit accepter comme inévitables les conflits entre le capital et le travail. De plus, elle ne doit plus avoir comme philosophie l'asservissement du travail aux seuls buts du capital. 2) Pour étudier la structure du travail et ses relations avec la capital, il serait bon de consulter les documents officiels tels que les constitutions, les chartes, les contrats et de découvrir comment ils ont évoluer. 3) Le contrôle exercé par les 《leaders 》 syndicaux reflète-t-il le fonctionnement d'une institution démocratique? Une grande quantité d'informations serait obtenue par une observation systématique des relations entre le meneur et ses subalternes. 4) Quels sont les moyens de communications développés ou utilisés par les représentants syndicaux? 5) Quels sont les conflits d'autorité causés par la fondation de groupes nationaux? Dans quelle mesure $\mathrm{la}$ bureaucratie influence-t-elle l'administration du syndicat? 6) Il y a très peu de recherche sur les problèmes administratifs rencontrés par le représentant syndical et sur la suffisance des procédures administratives. 7) Un autre domaine très dynamique consisterait à évaluer et à modifier les perceptions réciproques du capital et du travail.

Cet ouvrage de MacDonald résume et commente très bien la recherche sur l'autorité syndicale. Concis et bien étoffé, ce volume se doit d'intéresser les chercheurs dans le domaine des sciences humaines et sociales.

\section{C.-R. Giroux}

Inside a Sensitivity Training Groups, by T.R. Weschler \& J. Reisel, Institute of Industrial Relations. University of California, Los Angeles, Monogra Series: $4,134 \mathrm{pp}$.

Un drame humain, un drame social, un drame authentique, puisqu'il s'agit de l'impuissance d'un groupe à solutionner ses problèmes. Le groupe ne réussit même pas à s'entendre sur sa structure et sur un mode d'opération. Ce petit ouvrage est des plus captivants. Le lecteur suit l'évolution du groupe, ses tentatives de croissance, ses capitulations face aux conflits émotifs et aux attitudes négatives de certains membres. Le lecteur attend vainement un dénouernent de dernière heure. Le groupe capitule enfin, laissant le lecteur aux prises avec un profond sentiment de mélancolie. La croissance des membres a-t-elle avortée!

Le présent ouvrage décrit comment tout membre d'un groupe doit non seulement se comprendre objectivement, mais accepter ses propres déficiences avant de pouvoir entretenir des interrelations efficaces avec ses compagnons. Pendant longtemps l'on tenta de développer et d'entraîner les administrateurs et les gérants en leur enseignant les méthodes administratives, en augmentant leurs connaissances techniques, pour enfin se rendre à l'évidence que les résultats attendus étaient loin de se concrétiser parce que les individus se montraient incapables d'entretenir les contacts humains flexibles et exempts de conflits. La croissance et le développement d'un individu ne peut s'opérer qu'en fonction de ses contacts humains. Dans les cas extrêmes il faut avoir recours à une thérapie de groupe. Pourquoi ? Parce que c'est la seule façon de calibrer les réactions individuelles à un diapason commun, i.e. en fonction d'un comportement commun.

Plusieurs méthodes ont été mises au point pour provoquer la croissance de l'individu au sein du groupe. Trois méthodes sont surtout connues, celle du «Sensitivity Training - University of California », celle du \& Leaderless Group Discussion - Louisiana State University » et celle du «National Training Laboratory ». Les trois méthodes utilisent la technique non-directive de Rogers.

Le comportement du groupe se manifeste par les interactions entre les membres. Ces échanges individuels oscillent entre deux pôles, la subjectivité des intérêts et des problèmes personnels, et l'objectivité engendrée par la compréhension et l'acceptation des réactions des autres. Si chacun des membres du groupe réussit à se dégager de ses préoccupations personnelles, à comprendre et à accepter ses besoins, ses craintes et ses tendances, il se montrera alors plus réceptif envers ses camarades. plus compréhensif et enfin plus conciliant. Cette croissance individuelle limitera les comportements irrationnels des membres. 